\title{
Adaptive Natural Oscillator to Exploit Natural Dynamics for Energy Efficiency
}

\author{
Mahdi khoramshahi*, Rezvan Nasiri, Mohammad Shushtari, Auke Ijpeert, M. Nili Ahmadabadi \\ Cognitive Robotics Laboratory, School of ECE, College of Engineering, University of Tehran, Iran \\ Biorobotics Laboratory, School of Engineering, EPFL, Switzerland
}

\begin{abstract}
We present a novel adaptive oscillator, called Adaptive Natural Oscillator (ANO), to exploit the natural dynamics of a given robotic system. This tool is built upon the Adaptive Frequency Oscillator (AFO), and it can be used as a pattern generator in robotic applications such as locomotion systems. In contrast to AFO, that adapts to the frequency of an external signal, ANO adapts the frequency of reference trajectory to the natural dynamics of the given system. In this work, we prove that, in linear systems, ANO converges to the system's natural frequency. Furthermore, we show that this tool exploits the natural dynamics for energy efficiency through minimization of actuator effort. This property makes ANO an appealing tool for energy consumption reduction in cyclic tasks; especially in legged systems. We also extend the proposed adaptation mechanism to high dimensional and general cases; such as $n$-DOF manipulators. In addition, by investigating a hopper leg in simulation, we show the efficacy of ANO in face of dynamical discontinuities; such as those inherent in legged locomotion. Furthermore, we apply ANO to a simulated compliant robotic manipulator performing a periodic task where the energy consumption is drastically reduced. Finally, the experimental results on a 1-DOF compliant joint show that our adaptive oscillator, despite all practical uncertainties and deviations from theoretical models, exploits the natural dynamics and reduces the energy consumption.
\end{abstract}

Keywords: Energy efficiency, Natural dynamics, Cyclic task, Adaptive oscillator, Pattern generator

\section{INTRODUCTION}

Energy efficiency along with stability and adaptability are the ultimate goals in most robotic applications, especially in cyclic tasks such as pick-and-place [1] and locomotion [2]. In a classical view towards motion generation and control, energy consumption is considered as a cost which can be optimized using numerous model-based methods such as optimal control [3]. However, such model-based approaches cannot be simply applied to nowadays robotic systems with compliant elements, nonlinearities, and hybrid dynamics. On the other hand, model-free optimization techniques, such as reinforcement learning [4] and adaptive control [5], can be easily applied for energy efficiency. Nevertheless, these methods suffer from slow and unwarranted convergence behavior. However, for energy-efficiency, focusing on natural dynamics can simplify and facilitate such methods. As presented in this work, we do not consider the energy efficiency as a blind optimization problem, but rather as the result of an adaptation between motiongeneration and natural dynamics.

Broadly speaking, energy efficiency can be achieved using two different approaches: Natural Dynamics Modification (NDM see [6-9]) and Natural Dynamics Exploitation (NDE see [10-13]). While NDM methods aim the structure design of the robot, NDE approaches try to modify the motions in order to improve the energy efficiency. In other words, NDE methods include all types of motions generators which target energy consumption reduction —as an

${ }^{*}$ Corresponding author 
objective - in their tuning/adapting parameters. Inspiration from biology has been a prosperous approach in designing energy efficient gait patterns; see [10] where quadrupedal gait patterns extracted from similar biological structures lead to a lower cost. Imitation learning is also a useful approach toward stability and energy efficiency where, for instance, robots can learn to locomote by imitating the motion patterns of a walking human; see [16]. Furthermore, inspiration from simple passive mechanical systems, and understanding their underlying principles is also beneficial in generating such energy-efficient motion patterns; see [11] where bipedal gait patterns are generated from passive walkers. In [12], it is shown that using natural dynamics of a simple passive walker as the initial dynamics facilitates the learning process; see also [13] for the quadrupedal locomotion.

In design of gaits and rhythmic patterns, the frequency of oscillations is the key design parameter for motiongeneration. According to biology, the frequency of locomotion has a significant influence on the stride-length, locomotion speed, and energy consumption [14,15]. In this study, we present a novel adaptive oscillator for generating energy-efficient rhythmic patterns. Here, we assume that the periodic shape of the oscillations is given and only the frequency and the phase lag are left to optimization. We show that by exploiting the natural dynamics of the system, this adaptive oscillator converges to the natural frequency of the system which results in minimum energyconsumption.

The rest of this article is organized as follows. We quickly review the related works in the next section and we present the ANO in Section 3. To keep the analytical arguments as simple as possible, we focus on single-coordinated systems, and we study the stability, convergence, and optimality in Section 4.1 and Section 4.2. In Section 4.3, we generalize the ANO for systems with higher degrees of freedom; i.e., $n$-DOF serial manipulators. Simulations and experimental results are presented in Section 5 and Section 6. Discussions and conclusion are placed in the last sections.

\section{Related Works}

Based on our knowledge from biology, periodicity and smoothness are among the main properties of joint trajectories in locomotion. A sinusoidal joint trajectory is the simplest form that meets these properties and is the core of Phase Oscillators. Due to their simplicity, Phase Oscillators are extensively used in robotic applications [17-20]. Moreover, implementing Phase Oscillators using dynamical systems, such as Hopf and Van Der Pol oscillators [21], offers us stable limit cycles with advantageous properties such as smooth convergence behavior; see [22] for application in swimming robots.

The coupling capacity increases the applicability of a network of Phase Oscillators it terms of complex rhythmic pattern generations. This capacity is exploited in Central Pattern Generator (CPG) Networks where frequency, amplitudes, and coupling-strengths of a group of oscillators parameterize the motion-generation; see [23] for a survey on CPG-networks and their applications. The provided parameter space is often explored to satisfy objective functions such as stability, locomotion speed, and cost of transportation; for example, in [24] ZMP constraints are satisfied by proper selection of CPG parameters. This exploration -i.e. parameter search- has been carried out using different techniques such as optimal control [25], reinforcement learning [26], and genetic algorithms [27]. The simplicity of CPG-networks allows for interesting extensions and applications in legged locomotion; see [28] as an example where obstacle avoidance over rough terrains is achieved by including virtual model and reflex control to the CPG-network.

Recurrent Neural Networks (RNN) offer a similar approach to CPG-networks where instead of coupled oscillators, coupled neurons (e.g., Leaky integrator neurons) generate rhythmic patterns [29, 30]. However, the unnecessary complexity of the neuron-model makes this tool less applicable for motion generation in robotic applications where tractability of the learning is of particular interest. Dynamic Movement Primitive (DMP) method provides such tractability by learning a transformation from a stable motion, generated by a simple dynamics, to a desirable complex pattern [31].

Coupling to an external signal is another capability of Phase Oscillators that enables them to provide adaptive behavior. Due to the fundamental role of frequency in energy consumption, locomotion speed, and stability, the 
frequency-adaptation is extensively studied and applied in robotic systems [32, 33]; especially in gait assistance and rehabilitation devices [34-36]. As another example, [37] shows that a simple frequency adaptation, through phaseresetting, can improve the gait-stability. Furthermore, Adaptive Frequency Oscillator (AFO) model [38] is proposed to enable the oscillator to adapt its frequency and phase to an external trajectory. We complement this body of works by introducing $A N O$ which adapts its frequency to the natural frequency of a given system; in other words, an ANO exploits the system's natural dynamics which leads to energy-efficient motion-generation.

\section{Adaptive Natural Oscillator}

In robotic applications, such as legged locomotion, the energy efficiency is achieved by reshaping the desired trajectory according to system's dynamics; i.e., natural dynamics exploitation. In this work, we only focus on adapting the phase and the frequency of the desired trajectory and propose the following adaptive oscillator.

$$
\begin{cases}\dot{\omega} & =\varepsilon \omega F(t) \cos (\theta) \\ \dot{\theta} & =\omega \\ x_{d} & =A \cos (\theta)\end{cases}
$$

Similar to AFO [39], in this adaptive oscillator $\theta$ and $\omega$ are the phase and the frequency respectively and the adaptation speed is controlled by $\varepsilon$. The force applied by the controller/actuator $(F)$ is used as the sensory feedback for this oscillator while the oscillator's output $\left(x_{d}\right)$ is the desired trajectory. Fig. 1 shows how ANO works in this closed-loop fashion.

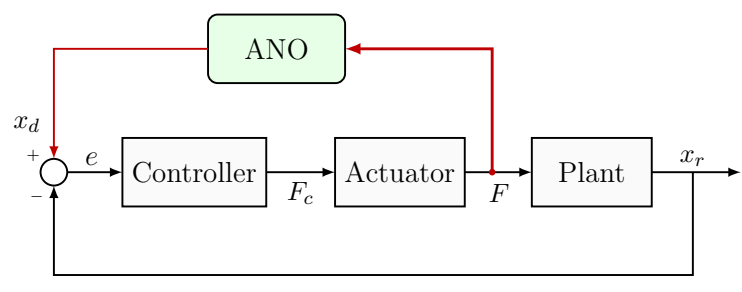

Fig. 1: Using an adaptive natural oscillator to exploit the natural dynamics. The oscillator is employed as a pattern generator, and the applied force is used as feedback.

By comparing ANO (Eq. 1) with AFO ([39]), it can be inferred that unlike AFO that exploits natural frequency of the target trajectory $(\Gamma)$, ANO exploits the natural frequency of the applied force $(F)$ which, consequently, leads to natural dynamics exploitation. While frequency convergence of ANO is similar to AFO, phase locking is slightly different. Using $\cos (\theta)$ (instead of $\sin (\theta)$ in AFO), ANO tries to create a $90^{\circ}$ phase lag between force $(F)$ and position $\left(x_{d}\right)$. This property of ANO is be explained further in Section 7.1.

\section{Mathematical Analysis}

We start this section by studying the frequency convergence along with phase locking behavior in ANO. Then, we show how ANO works towards energy efficiency, and finally, we extend the ANO idea to a general manipulator system. 


\subsection{Stability and Frequency Convergence Behavior}

Consider a simple mass-spring system. Having dynamics of the system, applied force for a certain trajectory is

$$
F=m \ddot{x}_{r}+k x_{r}
$$

where $x_{r}$ is the position of mass $(m) . k$ is the spring constant and $F$ is the force applied by the controller. To fully specify the dynamical system, as illustrated in Fig. 1, we should also consider the ANO equations (Eq. 1). For the sake of simplicity, we set the oscillations amplitude to one $(A=1)$. Using ANO output $\left(x_{d}\right)$ as the desired trajectory for the mass-spring system, and with perfect tracking assumption $\left(x_{r} \equiv x_{d}\right)$, we can calculate the applied force $(F)$ as follows:

$$
F=\left(k-m \omega^{2}\right) \cos (\theta)-m \dot{\omega} \sin (\theta)
$$

By defining $\omega_{n}=\sqrt{k / m}$, and substituting the applied force from Eq. 3 into frequency adaptation rule (Eq. 1), we have the following dynamical system.

$$
\begin{aligned}
& p \dot{\omega}=-\varepsilon \omega\left(\omega-\omega_{n}\right)\left(\omega+\omega_{n}\right) \cos ^{2}(\theta) \\
& p=1+\varepsilon m \omega \sin (\theta) \cos (\theta)
\end{aligned}
$$

With the assumption of slow adaptation $(\varepsilon<2 /|m \omega|)$, we can guarantee that $p>0$, where the stability of the system (Eq. 4) can be studied using Lyapunov Theorem and Barballat Lemma. Using the following Lyapunov candidate (Eq. 5), one can simply show that $\omega_{n}\left(-\omega_{n}\right)$ is stable for positive (negative) frequencies. This leaves the origin as an unstable equilibrium point.

$$
V=\frac{1}{2}\left(\omega \mp \omega_{n}\right)^{2}
$$

\begin{tabular}{|c|c|c|}
\hline Parameter & & Value \\
\hline Initial frequency & $\omega_{0}$ & $0.5 \mathrm{rad} / \mathrm{s}$ \\
\hline Amplitude & $A$ & $1 m$ \\
\hline Adaptation rate & $\varepsilon$ & 0.05 \\
\hline
\end{tabular}

To demonstrate ANO convergence behavior in simulation, a simple PID controller $\left(k_{p}=100, k_{i}=1\right.$, and $\left.k_{d}=10\right)$ is used to control the position of mass on the desired trajectory $\left(x_{d}\right)$ provided by ANO with parameters specified in Table. 1.

Adaptation results are depicted in Fig. 2a. First subplot shows that frequency of the oscillator converges to the natural frequency of the system $(\sqrt{k / m})$. Consequently, the applied force, illustrated in the second subplot, diminishes to zero. This behavior in the control effort ensures that natural dynamics are exploited. Last subplot shows that tracking performance, provided by the PID controller, is satisfactory.

To study the convergence behavior further, and to check the basins of attraction of the system, the previous simulation is repeated with different initial conditions $\left(\omega_{0}=\{-5,-1,1,5\}\right)$. Solutions of the system for these initial conditions are plotted in the phase plane; see Fig. 2b. It can be seen that solutions starting in positive (negative) frequencies converge to $\omega_{n}\left(-\omega_{n}\right)$. It is also interesting to note that solutions are modulated with $\cos ^{2}(\theta)$ as in Eq. 4 . Upper and lower profiles can be extracted by setting $\cos ^{2}(\theta)$ to zero or one in Eq. 4 .

$$
\left\{\begin{array}{l}
\cos ^{2}(\theta)=0 \Rightarrow \dot{\omega}=0 \\
\cos ^{2}(\theta)=1 \Rightarrow \dot{\omega}=-\varepsilon \omega\left(\omega-\omega_{n}\right)\left(\omega+\omega_{n}\right)
\end{array}\right.
$$

For the second profile, we have $p=1$ since $\sin (\theta)=0$. These profiles, along with the solutions, are plotted in Fig. $2 \mathrm{~b}$. Due to small error in tracking, solutions are not exactly placed between these profiles. 

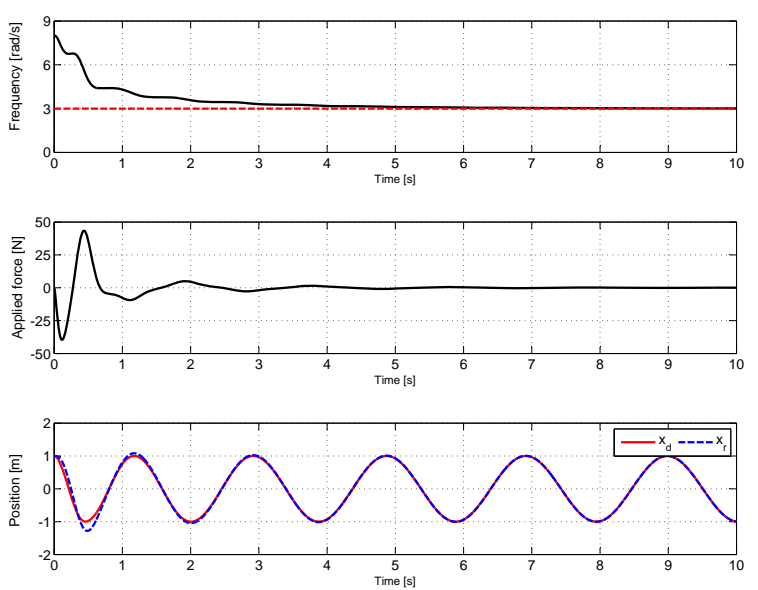

(a)

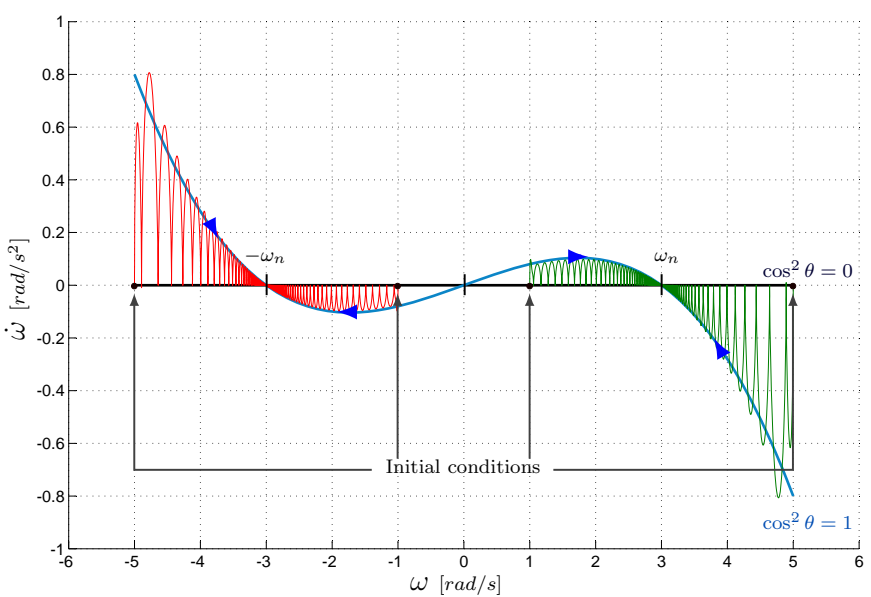

(b)

Fig. 2: (a) Adaptation result for the mass-spring system ( $m=1 \mathrm{~kg}$ and $k=9 \mathrm{~N} / \mathrm{m})$. (b) Convergence behavior of ANO for mass-spring system. Solutions are plotted for four different initial conditions $\left(\omega_{0}=\{-5,-1,1,5\}\right)$ with $\varepsilon=0.01$.

\subsection{Adaptation as the solution to a Minimization Problem}

It can also be shown that the proposed adaptive oscillator minimizes the following cost function.

$$
J(t)=\frac{1}{2} F^{2}(t)
$$

Using gradient decent method on the mentioned cost function yields:

$$
\dot{\omega}=-\lambda \nabla_{\omega} J(t)=-\lambda F(t)\left(\frac{\partial F}{\partial \omega}\right)
$$

By taking the partial derivative from Eq. 3, we have

$$
\dot{\omega}=2 m \lambda \omega F(t) \cos (\theta)
$$

By defining $\varepsilon=2 m \lambda$, we reach the adaptation method in Eq. 1. Given a predefined trajectory, $F^{2}$ and $\left|F \dot{x}_{r}\right|$ minimization are equivalent. Thus, under perfect-tracking assumption, ANO minimizes the instantaneous power consumption.

\subsection{Extended adaptive natural oscillator}

In this subsection, we extend the presented adaptation rule from a simple mass-spring system to a system with higher degrees of freedom and nonlinear dynamical equations. Consider a fully-actuated $n$-DOF serial manipulator with dynamics specified as follows:

$$
M(q) \ddot{q}+C(q, \dot{q})+G(q)=F
$$

In this equation of motion, $q$ and $\tau \in \mathbb{R}^{n}$ represent joints position and applied torque respectively. $M(q) \in \mathbb{R}^{n \times n}$ represents the mass matrix, $C(q, \dot{q}) \in \mathbb{R}^{n}$ denotes centrifugal and Coriolis forces, and $G(q) \in \mathbb{R}^{n}$ indicates the gravitational and passive forces. For a general periodic task, we consider the following parametrized reference trajectory for the end-effector.

$$
\left\{\begin{array}{l}
\dot{\theta}=\omega \\
X_{d}=R(\theta)
\end{array}\right.
$$

It can be seen that the phase $(\theta)$ of a unit harmonic oscillator, with frequency $\omega$, is mapped through an arbitrary but isomorphic transformation $(R)$ into a periodic desired trajectory $\left(X_{d}\right)$ for the manipulator end-effector. Here, we 
assume that the manipulator is non-redundant for this task. Interestingly, the desired torque for the desired trajectory $\left(X_{d}\right)$ can be expressed by the parameters of the unit harmonic oscillator $(\theta$ and $\omega)$ :

$$
\tau=\Psi(\theta) \dot{\omega}+\Upsilon(\theta) \omega^{2}+G(\theta)
$$

where $\Psi$ and $\Upsilon$ can be obtained using geometry of the desired trajectory $(R)$, the dynamics $(M$ and $C)$, and the forward kinematics of the manipulator; see Appendix. In accordance with the previous section, adaptation rule can be derived through the minimization of instantaneous torques. Henceforth, we consider the following quadratic cost function similar to Eq. 7.

$$
J=\frac{1}{2}\|\tau\|_{2}^{2}
$$

Using Eq. 12 and gradient descent method $(\dot{\omega}=-\lambda \partial J / \partial \omega)$ yields the generalized rule for frequency adaptation:

$$
\dot{\omega}=-\epsilon \omega \tau^{T} \Upsilon(\theta)
$$

The extracted adaptation rule, is a function of dynamical equations of the manipulator ( $\Upsilon$ ). However. having $\Upsilon(\theta)$ precomputed, the general adaptation rule is still simple compared to the previous case (Eq. 1). Interestingly, Eq. 14 can be used to extract an adaptation rule for a linear 1-dof system with an arbitrary reference path (i.e., $R(\theta))$ as follows.

$$
\begin{cases}x_{d} & =R(\theta) \\ \dot{\theta} & =\omega \\ \dot{\omega} & =-\varepsilon \omega F(t) \partial^{2} R(\theta) / \partial \theta^{2}\end{cases}
$$

Further assumption of sinusoidal trajectories (i.e., $R(\theta)=\cos (\theta)$ ) yields again the original ANO in Eq. 1. Moreover, adaptation rules for a non-linear 1-dof system with an arbitrary reference path can be extracted from Eq. 14. In [40], we presented preliminary results for exploitation of such nonlinear dynamics, especially nonlinear compliances.

\section{Simulations}

In this section, to show the applicability of our approach, we consider more complex scenarios with dynamical discontinuity, nonlinearity, and higher dimensionality; i.e., a hopper leg and a 2-DOF compliant manipulator. Numerical simulations for these system are carried out with MATLAB/Simulink/Simmech [41] using ode45 solver.

\subsection{Hopper leg}

Hopper systems, as a tool in robotics to understand the principals of running, can be traced back to 80's; see [42]. Therefore, providing adaptive mechanisms that exploits its natural dynamics can be beneficial to design energy efficient running systems. Consider a simple vertical hopper leg illustrated in Fig. 3a. This hopper leg consists of a prismatic joint (actuator), a mass-less leg, and a parallel spring. Despite its simplicity compared to a full-body legged system, the natural dynamics of this system, due to its hybrid dynamics, is not as straightforward as linear systems. During the stance phase, mass-spring dynamics and during the flight phase, ballistic dynamics govern the system. The transition between these two dynamics is determined when the spring is at its rest-length. Note that the actuator can only exert force during ground contact. The task for this hopper is to move on a sinusoidal trajectory with amplitude of $0.1 \mathrm{~m}$ and an offset equal to spring rest length $\left(l_{0}=1 \mathrm{~m}\right)$. However, frequency of this oscillation is left for ANO to be adapted.

Fig. 3b shows the adaptation behavior for the hopper leg. First subplot shows that frequency, on average, converges to $12.4 \mathrm{rad} / \mathrm{s}$ which substantially differs from $\sqrt{\mathrm{k} / \mathrm{m}}$ in this system. Importantly, small ripples insinuate that sinusoidal trajectory is not perfectly consistent with the natural dynamics of this system. Second subplot shows the control effort. It can be seen that the magnitude of applied force is reduced significantly. It is also interesting to see that the system quickly learns not to exert negative force; it is intuitive to only use positive forces for hopping in a compliant system. The last subplot shows the tracking performance. Tracking performance improves along with adaptation. This is again intuitive that tracking along natural dynamics is more precise for imperfect controllers such as PID controller. Negligible error around apex is inevitable since there is no actuation during the flight phase. Interestingly, according to unreported results, the converged frequency is less sensitive to the spring constant compared to the amplitude of oscillators $(A)$. 


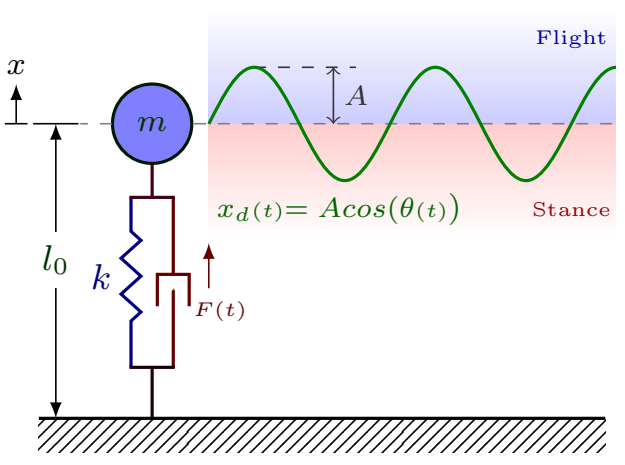

(a)
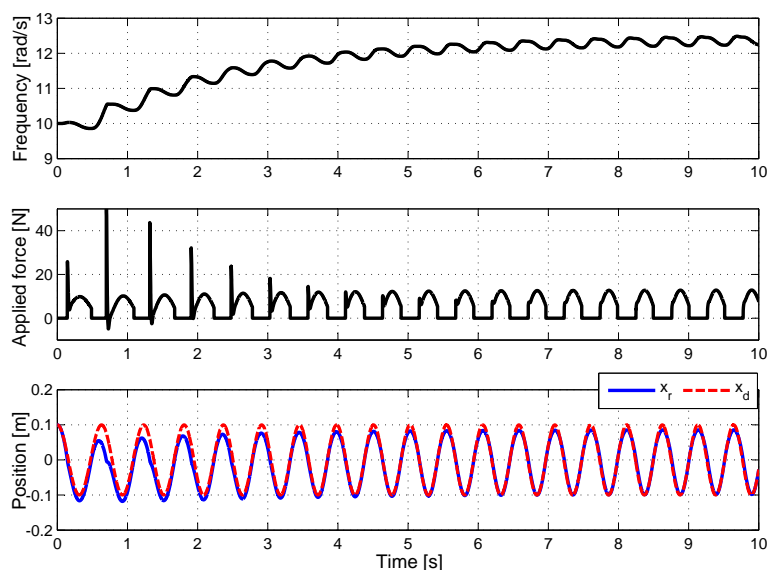

(b)

Fig. 3: (a) A hopper robot with a parallel compliant in the leg. Mass of the hopper $(\mathrm{m})$ is $0.5 \mathrm{~kg}$, and for spring, damper, and gravity, we have $k=1 \mathrm{~N} / \mathrm{m}, b=0.1 \mathrm{Ns} / \mathrm{m}$, and $g=9.81$. (b) Adaptation result for hopper leg. PID gains are $k_{p}=500, k_{i}=100$, and $k_{d}=50$, and for adaptation $\epsilon=0.01$.

\subsection{Manipulator with extended ANO}

In this simulation, using the extended ANO, we exploit the natural dynamics of a non-redundant 2-DOF manipulator (Fig. 4). We use operational space control presented in [43] to move the the end-effector on a circular path. This path/task is parameterized as follows:

$$
\Gamma(\theta)=\left\{\begin{array}{l}
x_{d}=r \cos (\theta)+x_{0} \\
y_{d}=r \sin (\theta)+y_{0}
\end{array}\right.
$$

where $\theta$ is the phase of desired path and $x_{d}, y_{d}$ are the outputs of extended ANO. The parameters of desired path are $r=0.5 \mathrm{~m}, x_{0}=0 \mathrm{~m}$, and $y_{0}=1 \mathrm{~m}$. Adaptation rate $(\epsilon)$ is set to 0.06 , and frequency is initialized on $\omega_{0}=0.05 \mathrm{rad} / \mathrm{s}$. The robot is set in the horizontal plane; i.e., there is no gravity acceleration. The initial position of the end effector is considered on the reference path and the initial joint velocity is set to zero. The results of this simulation are illustrated in Fig. 5. Frequency of the oscillations, on average, is adapted to 5rad/s; see Fig. 5a. The ripples suggest that the desired path is not perfectly consistent with the natural dynamics of the system. Energy consumption for each cycle is illustrated in Fig. 5b. Drastic improvement in the energy efficiency suggests that natural dynamics of the system are exploited. During adaptation the cost function converges from $3729 \mathrm{~N}^{2} \mathrm{~m}^{2}$ to $242 \mathrm{~N}^{2} \mathrm{~m}^{2}$ (about $93 \%$ improvement w.r.t initial condition). Interestingly, the adaptation exhibit an damped exponential behavior where the energy consumption is drastically decreased after a few cycles; i.e., $80 \%$ after 5 cycles. The applied torque to each joint is plotted in Fig. 5c. Being bounded, these torque profiles converge to a steady-state behavior. Finally, Fig. 5d shows that tracking performance improves in the course of adaptation.

By repeating this simulation in the vertical plane (i.e., considering gravity acceleration), similar results were achieved. The frequency, on average, converged to $4.5 \mathrm{rad} / \mathrm{s}$. Also the initial and the final values of the cost function were $3447 \mathrm{~N}^{2} \mathrm{~m}^{2}$ and $255 \mathrm{~N}^{2} \mathrm{~m}^{2}$ respectively (about $92 \%$ improvement w.r.t initial condition). 


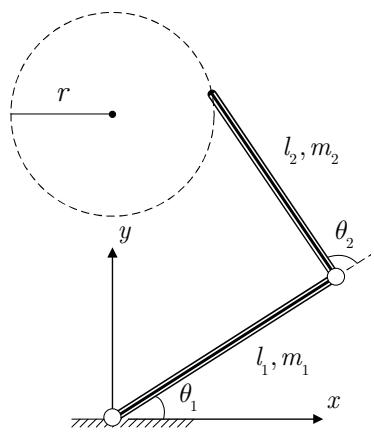

Fig. 4: A 2-DOF planner system with $l_{1}=l_{2}=1 \mathrm{~m}$, and $m_{1}=m_{2}=1 \mathrm{Kg}$. Inertias are calculated based on geometry of the links. The compliance rest-length and stiffness for both of the joints are $d_{1}=d_{2}=0 \mathrm{rad}$ and $k_{1}=k_{2}=20 \mathrm{Nm} / \mathrm{rad}$ respectively.

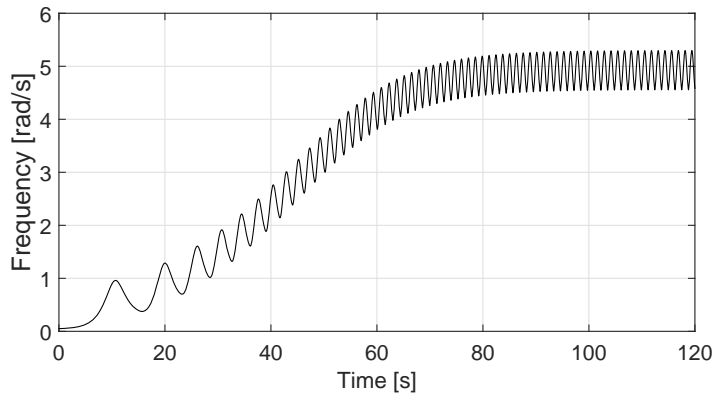

(a)

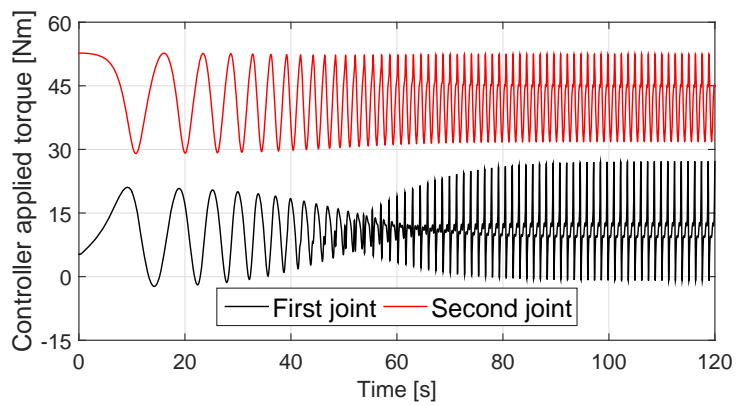

(c)

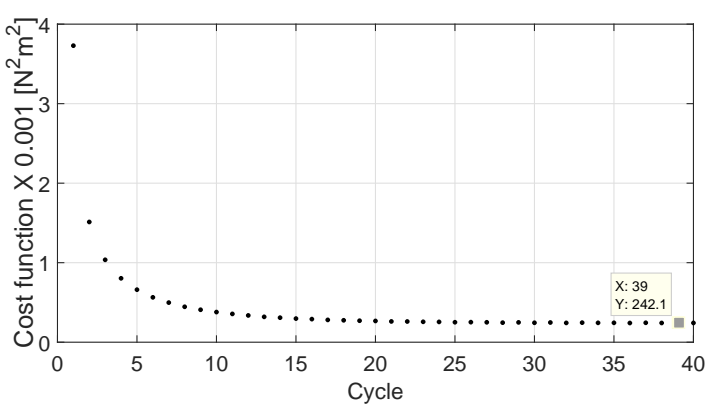

(b)

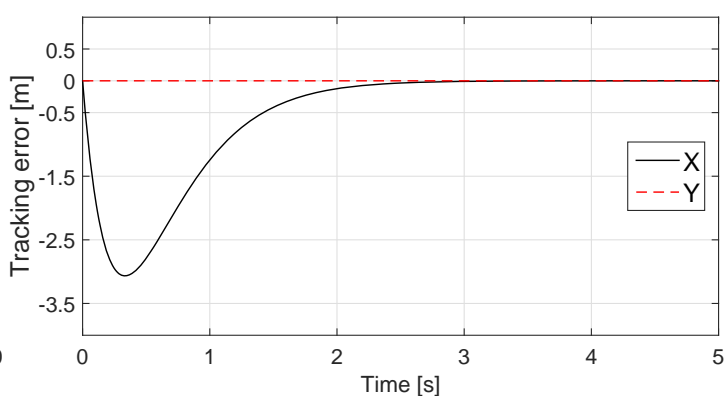

(d)

Fig. 5: Natural dynamics adaptation in the 2-DOF manipulator. (a) Frequency of the task. (b) The cost function is sum of integrate of squared applied torques for each cycle. Note that the cost function after 60 period is not plotted due to the lack of new information. (c) Controller applied toques. (d) End effector racking error in $x$ and $y$ directions. In this figure, in order to have a better vision, we zoom on the interval between $t=0 s$ and $t=5 s$. 


\section{Experimental Results}

To check the validity of the presented adaptation method in practice, we designed a 1-DOF revolute joint with a variable compliance; see Fig. 6a. In this setup, the revolute joint is actuated by a dc-motor (with $12 \mathrm{~V}$ terminal voltage and $0.5 \mathrm{Nm}$ maximum torque). The motor is non-back-drivable and its no-load current is about $100 \mathrm{~mA}$. The joint is controlled using PWM method. The compliance has been approximated to be linear (see Eq. 3-5 in [44]) with $K=0.42 \mathrm{Nm} / \mathrm{rad}$ for the initial strain $\left(S=S_{0}\right)$. The revolute joint's compliance $(K)$ is tunable using a stepper motor through varying the strain $(S)$ of linear springs; see [45] and [9] for similar designs. In this experiment, aforementioned properties of the motors along with frictions and other uncertainties suggest a considerable deviation from the ideal cases in the simulation section.

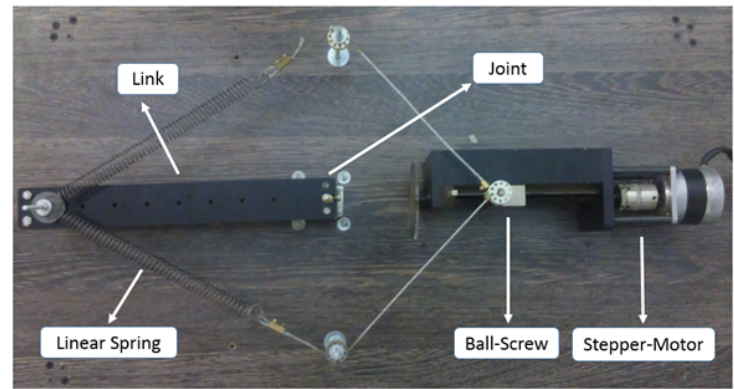

(a)

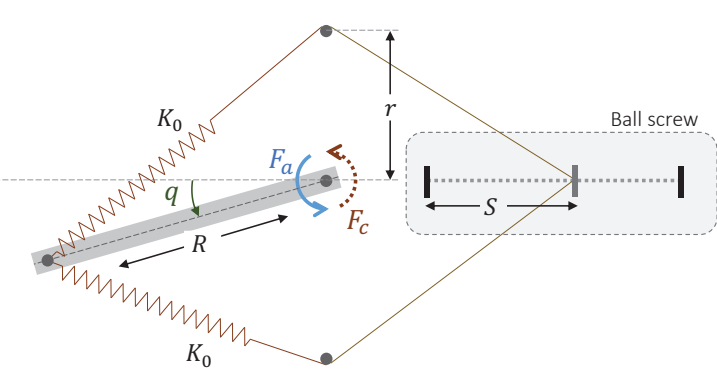

(b)

Fig. 6: (a) Experimental setup. The dc-motor is mounted on the other side of the table and cannot be seen in this view. The linear springs act as a parallel rotational spring at the joint. (b) Schematic of the compliance mechanism. $F_{a}\left(F_{c}\right)$ is the force applied at the joint by the dc-motor (parallel compliance mechanism). $R$ is the link length, $r$ is the distance between the revolute joint and the fixed side of linear spring, and $K_{0}$ is stiffness of the linear springs. $S$ is the strain of the linear springs which is changed by the stepper motor.

Fig. $6 \mathrm{~b}$ illustrates the schematic of setup structure. The desired trajectory is generated using the presented adaptation rule in Eq. 1. Convergence rate $(\epsilon)$ is hand-tuned to 10 for this experiment, and real joint position $(q)$ is provided by an encoder. The controller command $\left(F_{a}\right)$, instead of the actual applied force, is used for adaptation. We discuss this alternative in Section 7.3. Similar to [46], for the velocity control of the dc-motor (using PWM), a PI controller with $K_{p}=1$ and $K_{i}=0.2$ is used. Also, similar to the simple mass-spring system presented in Section 4.1, the reference sinusoidal trajectory ( $20 \mathrm{deg}$ amplitude) is generated by adaptive natural oscillator.

The overall performance of our adaption method is presented in Fig. 6. Fig. 7a shows convergence of the frequency to the optimal value; i.e., approximately $6.3 \mathrm{rad} / \mathrm{s}$. To study the energy consumption of the system in the course of adaptation, we use the average input power. Having a switching terminal voltage (between 0 and 12), this measure is proportional to the input current. Interestingly, in a dc-motor, the applied torque is also proportional to the input current. Therefore, the torque-minimization property of our method should lead to a minimization behavior in the average input power. This fact can be seen in Fig. $7 \mathrm{~b}$ where frequency adaption leads to a drastic decline in power consumption (about $44 \%$ improvement). Due to damping, frictions, non-perfect actuator, and other uncertainties, zero energy consumption cannot be obtained. The RMS tracking error, illustrated in Fig. 7c, implies that, tracking error is reduced during the adaptation. Finally, we investigate our adaption method under different compliance coefficients. Here, we repeat the previous experiment (Fig. 7a) with different joint stiffnesses. In each case, the frequency is adapted to the corresponding optimal value; see Fig. $7 \mathrm{~d}$ where higher stiffness leads to higher optimal frequency. 


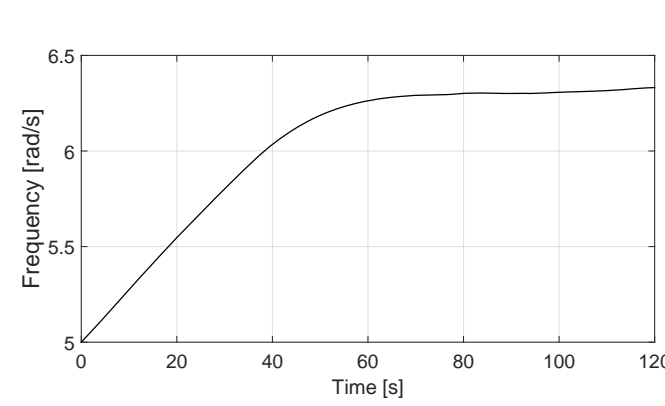

(a)

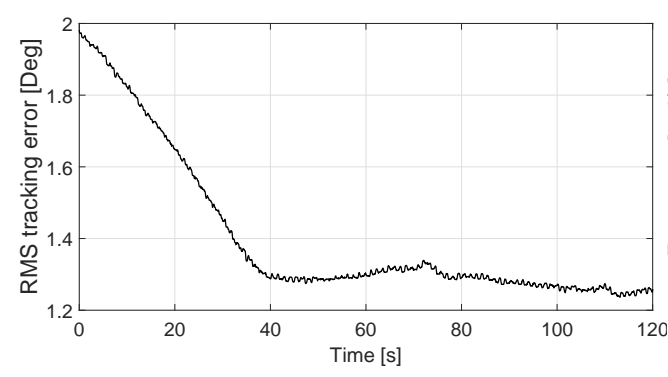

(c)

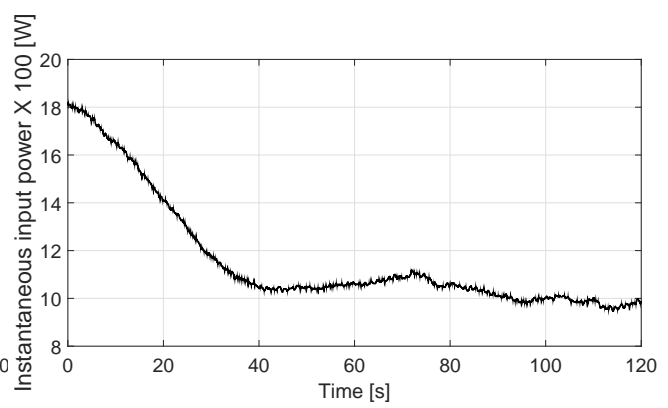

(b)

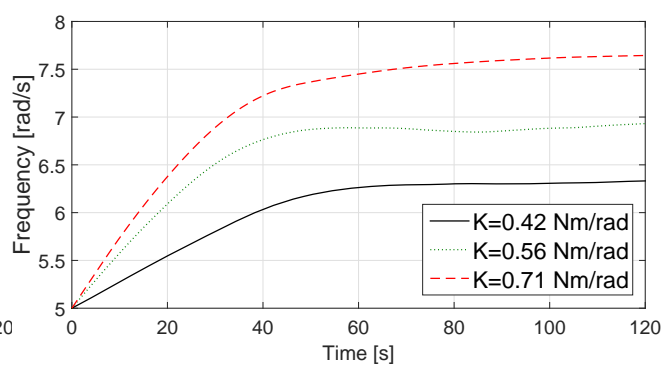

(d)

Fig. 7: Overall performance of the frequency adaptation for 1-DOF revolute joint. (a) The frequency adaptation shows a convergent behavior. (b) Average input power is reduced by adapting the compliance. (c) The tracking error RMS divided by averaging time. (d) Frequency convergence for different compliance values. It is important to note that all figures are averaged using a moving window of 5000 samples (sampling rate is 162 sample/s).

\section{Discussions}

In the following, we discuss other properties of the presented tool such as phase-locking behavior. We also emphasize on the importance of compliant elements in periodic tasks in order to achieve energy efficient cyclic motion. Moreover, using feedback of controller command instead of force signal as an alternative approach is discussed. In addition, we present a possible modification to the current method. Finally, robustness analysis of the extended adaptation rule (EANO) in face of unmodeled dynamics and model uncertainties are presented.

\subsection{Force-velocity synchronization}

Besides frequency convergence in ANO, it is also important to study the phase locking behavior of this oscillator from the energy consumption point of view. Given the fact that most actuators cannot recycle the negative work (i.e., where the applied force and the joint velocity have opposite signs), energy consumption is often calculated as:

$$
W=\int|F(t) v(t)| d t
$$

where $F(t)$ and $v(t)$ are the applied force and the joint velocity respectively. By synchronizing applied force and joint velocity, negative work will be minimized which could potentially lead to energy consumption minimization. Interestingly, this synchronization takes place in ANO. As we mentioned in Section 3, ANO tries to lock its output $\left(x_{d}\right)$ with $90^{\circ}$ lag to the applied force $(F)$. Adding the fact that in sinusoidal trajectories, there is also a $90^{\circ}$ phase lag between position and velocity, it could be concluded that ANO tries to lock the velocity $\left(\dot{x}_{d}\right)$ with the applied force $(F)$. Therefore, as ANO exploits the natural frequency of applied force, it also synchronizes velocity with force which eventually leads to natural dynamics exploitation; i.e., energy consumption reduction. 


\subsection{Periodic motions need compliance}

According to Eq. 4, the dynamical equation of ANO in the mass-spring system has two stable equilibrium points $\left( \pm \omega_{n}\right)$. These equilibrium points are functions of compliance as $\omega_{n}= \pm \sqrt{k / m}$. Therefore, $k=0$ results in zero stable equilibrium point $\left(\omega_{n}=0\right)$. Such dynamics imply that compliant elements are crucial to have adaptive behaviors toward energy efficient cyclic motions. The same was observed for the manipulator simulation and the experimental setup, where without compliant elements the frequency converged to zero. Accordingly, it is inferred that each optimum cyclic motion have a compatible compliance element. Consequently, an inverse problem comes to mind where in our previous works [47, 48], we provided methods to find the optimal compliance for a given periodic motion.

\subsection{Force sensor}

In the closed-loop system in Fig. 1, it is possible to use the controller output $\left(F_{c}\right)$ instead of the actuator force $(F)$. In this case, ANO exploits the natural dynamics of the system along with the actuator (i.e., augmenting the actuator to the plant). Since force sensors are expensive, imprecise, and noisy, using controller output is more practical. Moreover, since we are concerned about the total power consumption (plant and actuator), using controller output is more reasonable. In fact, we used the controller output for the adaptation in our experimental setup (Section 6) which leads to a drastic energy consumption reduction.

\subsection{Robustness against unmodeled dynamics}

According to Section 4.3, extracting the exact adaptation rule for an $n$-DOF manipulator requires knowledge about the dynamics of the system. This assumption can be seen as a disadvantage of the presented approach. Here, we study the robustness of our method to deviation from the nominal cases presented in Section 5.2. Two different types of uncertainties (i.e., mismatched dynamical parameters) are investigated: (1) a mismatch in the inertia matrix of the robot, and (2) a mismatched in the damping constant. The results are presented in Table. 2 and Table. 3 respectively. These results show minor deviations form the optimal point for the frequency and the energy consumption, and the robustness of the method (i.e., the EANO) to significant deviation from the nominal model.

Table 2: Effect of inertia deviation from nominal model on converged frequency and minimum cost function.

\begin{tabular}{l|ccccccccc} 
Inertia deviation $(\%)$ & -20 & -15 & -10 & -5 & 0 & 5 & 10 & 15 & 20 \\
\hline Frequency deviation (\%) & -10.2 & -7.5 & -4.8 & -2.2 & 0 & 2.7 & 5.2 & 7.5 & 9.8 \\
Cost function deviation (\%) & 9 & 6.3 & 3.7 & 1.6 & 0 & -2.2 & -4.5 & -5.3 & -6.6
\end{tabular}

Table 3: Effect of unknown damper coefficient on converged frequency and minimum cost function.

\begin{tabular}{l|ccccccccc} 
Damper coefficient $(\mathrm{Nms} / \mathrm{rad})$ & 0 & 0.25 & 0.5 & 0.75 & 1 & 1.25 & 1.5 & 1.75 & 2 \\
\hline Frequency deviation $(\%)$ & 0 & 0.4 & 0.8 & 1 & 1.2 & 1.2 & 1 & 0.8 & 0.6 \\
Cost function deviation $(\%)$ & 0 & 0.2 & 0.5 & -0.3 & 0 & 0.1 & 0.2 & 0.3 & 1.7
\end{tabular}

\subsection{Shaping the limit cycle}

Inconsistency between the shape of the reference path and natural dynamics of the robot is an undesirable factor which has a negative impact on the energy efficiency. For instance, in a mass-spring system with linear compliance, frequency adaptation for any non-sinusoidal preference path cannot result in fully natural dynamics exploitation (i.e., zero applied force as shown in Fig. 2a). Such inconsistencies were observed in Fig. 3b and Fig. 5a where there are nonvanishing ripples in the frequency adaptation. Our extended adaptation rule allows for modifications in the reference path. However, finding an optimal solution is an interesting problem which lies out of the scope of this work. For this purpose, generic and parametrized oscillators can be used to adapt not only the frequency, but also the shape of the limit cycle to the natural dynamics of the system [49]. For example, Dynamic Movement Primitives (DMP) [50] is a 
powerful tool which employs a generic oscillator at the core, and allows for many possible transformation to generate task-relevant motions [51]. Using nonlinear transformation as presented in [52] and [53] to create more sophisticated trajectories for ANO is also an interesting study. In [49], we present an adaptive nonlinear-oscillator that can produce dynamics-consistent motions for a single-joint system.

\section{Conclusions}

In this paper, we presented Adaptive Natural Oscillator (ANO), a tool for natural dynamics exploitation. This tool reduces the energy consumption by means of adapting the frequency and phase of the cyclic input trajectory according to the natural dynamics of the system; see Fig.2a, 3b, 5d, and 7b. While the adaptation rules in ANO and AFO are structurally similar, their feedback signal, and as a result, their adaptation goals are different. In ANO, we used applied force as the feedback for the oscillator; see Fig.1. By exploiting the natural frequency of the applied force, ANO is able to exploit the natural dynamics of the system. Using Lyapunov stability theorem, we proved the stability for the mass-spring system, and we studied the convergence behavior of the system in theory (Section 4.1) and in simulation (Section 5). Moreover, for the mass-spring case, we showed that this adaptive tool minimizes the instantaneous applied force (Section 4.2) which could potentially lead to energy consumption reduction (Section 7.1).

Even though a compliant hopper leg, compared to most robotic models, is a simple model (Section 5.1), but hybrid dynamics make this system complex enough from natural dynamics point of view. For this system, ANO adapted the frequency of a sinusoidal input trajectory in order to reduce the applied force. However, unlike mass-spring system, the applied force did not converge to zero. From this result, we can conclude that sinusoidal trajectories are not consistent with the natural dynamics of this system. Unreported simulations suggest that the optimal frequency is more sensitive to the task amplitude rather than the spring constant.

We showed that by extending the adaptation mechanism to higher dimensions (i.e., higher degrees of freedom), natural dynamics of robotic manipulators can also be exploited systematically. We proved that by incorporating the kinematics and dynamics of a generic manipulator, a simple adaption rule can still be derived; i.e., the Extended Adaptive Natural Oscillator (EANO) (Section 4.3). Simulation of this case illustrated the efficacy of our tool for complex systems where the energy consumption of a robotic manipulator, for performing a cyclic task, was reduced drastically; about 93\% compared to initial frequency (Fig.7d). Moreover, in order to analyze behavior of the presented method in real world, we adapted frequency of the reference sinusoidal trajectory for a 1-DOF revolute joint (Section 6). The results of this experiment strongly support our mathematics and show applicability of the presented method in practice in terms of frequency convergence (Fig.7d) and energy consumption reduction (Fig.7b).

\section{Acknowledgment}

We would like to thank University of Tehran for providing support for this work. Authors would also like to thank Amir Sarabi and Hamed Jalali for their comments on this article. 


\section{Appendix}

In manipulator systems, the task $\left(X \in \mathbb{R}^{m}\right)$ is a function of joint positions $\left(q \in \mathbb{R}^{n}\right)$ and the jacobian matrix is defined as $J=\partial X / \partial q$. Here, we assume that the controller can satisfy the perfect tracking assumption $\left(X \equiv X_{d} \equiv\right.$ $R(\theta)$ ). According to Eq. 11, the desired path $\left(X_{d}=R(\theta)\right)$ in the task space is a function of $\theta$. Also if $n \geq m$, there exists at least a periodic solution for the inverse kinematic. Specially here, we assume that $n=m$. Utilizing such inverse kinematics, we can conclude that $q$ and consequently $J$ are functions of $\theta$. Therefore, we have the following properties.

$$
\begin{aligned}
& \dot{X}=J \dot{q} \rightarrow \dot{q}=J^{-1} \dot{X} \rightarrow \dot{q}=L(\theta) \dot{\theta} \\
& L(\theta)=J^{-1}(\theta) \frac{\partial R(\theta)}{\partial \theta} \\
& \ddot{X}=\dot{J} \dot{q}+J \ddot{q} \rightarrow \ddot{q}=J^{-1}(\ddot{X}-\dot{J} \dot{q}) \rightarrow \\
& \ddot{q}=T(\theta) \dot{\theta}^{2}+L(\theta) \ddot{\theta} \\
& T(\theta)=J^{-1}(\theta)\left(\frac{\partial^{2} R(\theta)}{\partial \theta^{2}}-\frac{\partial J(\theta)}{\partial \theta} L(\theta)\right)
\end{aligned}
$$

According to [54], the total kinetic energy of the manipulator could be obtained as $K=0.5 \dot{q}^{T} M(q) \dot{q}$. Regardless of damping effects in the system, the centrifugal and Coriolis forces (i.e., $C(q, \dot{q}))$ could be calculated as

$$
C(q, \dot{q})=\left.\frac{d}{d t} \frac{\partial K(q, \dot{q})}{\partial \dot{q}}\right|_{\ddot{q}=0}-\frac{\partial K(q, \dot{q})}{\partial q} K(q, \dot{q})
$$

As we mentioned before, the angular position and velocity of each joint of the manipulator is a function of $\theta$. Applying some algebraic manipulations we have:

$$
C(q, \dot{q})=H(\theta) \dot{\theta}^{2}
$$

where $h_{i}(\theta)$ (i.e., the $i$ th element of $H(\theta)$ ) could be obtained from the following equation.

$$
h_{i}=2 \sum_{j=1}^{n}\left(\left(\frac{\partial m_{i j}}{\partial q}\right)^{T} L(\theta) l_{i}(\theta)\right)-\frac{1}{2} L(\theta)^{T} \frac{\partial M(q)}{\partial q_{i}} L(\theta)
$$

In the presented equation $m_{i j}$ represents an element of $M(q)$. Finally, by substituting $(\dot{\theta}, \ddot{\theta})$ with $(\omega, \dot{\omega})$, replacing $\ddot{q}$ from Eq. 19, and replacing $C(q, \dot{q})$ from Eq. 20 in Eq. 10 the manipulator's applied forces could be represented as:

$$
\tau=\underbrace{M(\theta) L(\theta)}_{\Psi(\theta)} \dot{\omega}+\underbrace{(M(\theta) T(\theta)+H(\theta))}_{\Upsilon(\theta)} \omega^{2}+G(\theta)
$$

Finally, $\tau$ can be represented in the following format.

$$
\tau=\Psi(\theta) \dot{\omega}+\Upsilon(\theta) \omega^{2}+G(\theta)
$$




\section{References}

[1] M. Pellicciari, G. Berselli, F. Leali, A. Vergnano, A method for reducing the energy consumption of pick-and-place industrial robots, Mechatronics 23 (3) (2013) 326-334.

[2] C. J. Walsh, D. Paluska, K. Pasch, W. Grand, A. Valiente, H. Herr, Development of a lightweight, underactuated exoskeleton for loadcarrying augmentation, in: Robotics and Automation, 2006. ICRA 2006. Proceedings 2006 IEEE International Conference on, IEEE, 2006, pp. 3485-3491.

[3] A. E. Bryson, Applied optimal control: optimization, estimation and control, CRC Press, 1975.

[4] R. S. Sutton, A. G. Barto, Reinforcement learning: An introduction, Vol. 1, MIT press Cambridge, 1998.

[5] K. J. Åström, B. Wittenmark, Adaptive control, Courier Corporation, 2013.

[6] S. H. Collins, M. B. Wiggin, G. S. Sawicki, Reducing the energy cost of human walking using an unpowered exoskeleton, Nature 522 (7555) (2015) 212-215.

[7] B. Vanderborght, B. Verrelst, R. Van Ham, M. Van Damme, D. Lefeber, B. M. Y. Duran, P. Beyl, Exploiting natural dynamics to reduce energy consumption by controlling the compliance of soft actuators, The International Journal of Robotics Research 25 (4) (2006) $343-358$.

[8] N. Schmit, M. Okada, Optimal design of nonlinear springs in robot mechanism: simultaneous design of trajectory and spring force profiles, Advanced Robotics 27 (1) (2013) 33-46.

[9] B. Vanderborght, A. Albu-Schäffer, A. Bicchi, E. Burdet, D. G. Caldwell, R. Carloni, M. Catalano, O. Eiberger, W. Friedl, G. Ganesh, et al., Variable impedance actuators: A review, Robotics and Autonomous Systems 61 (12) (2013) 1601-1614.

[10] Y. Fukuoka, K. Fukino, Y. Habu, Y. Mori, Energy evaluation of a bio-inspired gait modulation method for quadrupedal locomotion, Bioinspiration \& biomimetics 10 (4) (2015) 046017.

[11] S. Collins, A. Ruina, R. Tedrake, M. Wisse, Efficient bipedal robots based on passive-dynamic walkers, Science 307 (5712) (2005) 10821085 .

[12] R. L. Tedrake, Applied optimal control for dynamically stable legged locomotion, Ph.D. thesis, Massachusetts Institute of Technology (2004).

[13] H. J. Bidgoly, A. Vafaei, A. Sadeghi, M. N. Ahmadabadi, Learning approach to study effect of flexible spine on running behavior of a quadruped robot, in: Proceeding of International Conference on Climbing and Walking Robots, Vol. 2010, 2010, pp. 1195-1201.

[14] D. Grieve, R. J. Gear, The relationships between length of stride, step frequency, time of swing and speed of walking for children and adults, Ergonomics 9 (5) (1966) 379-399.

[15] N. C. Heglund, C. R. Taylor, Speed, stride frequency and energy cost per stride: how do they change with body size and gait?, Journal of Experimental Biology 138 (1) (1988) 301-318.

[16] J. Nakanishi, J. Morimoto, G. Endo, G. Cheng, S. Schaal, M. Kawato, Learning from demonstration and adaptation of biped locomotion, Robotics and Autonomous Systems 47 (2) (2004) 79-91.

[17] P. New, C. Wheeler, T. G. Sugar, Robotic hopper using phase oscillator controller, in: ASME 2014 International Design Engineering Technical Conferences and Computers and Information in Engineering Conference, American Society of Mechanical Engineers, 2014, pp. V05BT08A068-V05BT08A068.

[18] T. G. Sugar, A. Bates, M. Holgate, J. Kerestes, M. Mignolet, P. New, R. K. Ramachandran, S. Redkar, C. Wheeler, Limit cycles to enhance human performance based on phase oscillators, Journal of Mechanisms and Robotics 7 (1) (2015) 011001.

[19] T. G. Sugar, S. Redkar, Bioinspired controller based on a phase oscillator, in: Converging Clinical and Engineering Research on Neurorehabilitation II, Springer, 2017, pp. 1371-1376.

[20] Y. Farzaneh, A. Akbarzadeh, A. A. Akbari, New automated learning cpg for rhythmic patterns, Intelligent Service Robotics (2012) 1-9.

[21] J. De la Fuente, T. G. Sugar, S. Redkar, Nonlinear, phase-based oscillator to generate and assist periodic motions, Journal of Mechanisms and Robotics 9 (2) (2017) 024502.

[22] C. Zhou, K. Low, Design and locomotion control of a biomimetic underwater vehicle with fin propulsion, IEEE/ASME Transactions on Mechatronics 17 (1) (2012) 25-35.

[23] A. J. Ijspeert, Central pattern generators for locomotion control in animals and robots: a review, Neural Networks 21 (4) (2008) 642-653.

[24] J. Or, A hybrid CPG-ZMP control system for stable walking of a simulated flexible spine humanoid robot, Neural Networks 23 (3) (2010) $452-460$.

[25] C. Tang, S. Ma, B. Li, Y. Wang, A self-tuning multi-phase cpg enabling the snake robot to adapt to environments, in: 2011 IEEE/RSJ International Conference on Intelligent Robots and Systems, IEEE, 2011, pp. 1869-1874.

[26] H. Benbrahim, J. A. Franklin, Biped dynamic walking using reinforcement learning, Robotics and Autonomous Systems 22 (3) (1997) 283-302.

[27] C. Liu, Q. Chen, D. Wang, Cpg-inspired workspace trajectory generation and adaptive locomotion control for quadruped robots, IEEE Transactions on Systems, Man, and Cybernetics, Part B (Cybernetics) 41 (3) (2011) 867-880.

[28] M. Ajallooeian, S. Pouya, S. Gay, A. Tuleu, A. Sprowitz, A. Ijspeert, Towards modular control for moderately fast locomotion over unperceived rough terrain, in: Dynamic Walking 2013, no. EPFL-POSTER-185884, 2013.

[29] B. A. Pearlmutter, Learning state space trajectories in recurrent neural networks, Neural Computation 1 (2) (1989) $263-269$.

[30] S. Townley, A. Ilchmann, M. G. Weiß, W. McClements, A. C. Ruiz, D. H. Owens, D. Pratzel-Wolters, Existence and learning of oscillations in recurrent neural networks, IEEE Transactions on Neural Networks 11 (1) (2000) 205-214.

[31] S. Schaal, J. Peters, J. Nakanishi, A. Ijspeert, Learning movement primitives, in: Robotics Research, Springer, 2005 , pp. 561-572.

[32] T. Nachstedt, C. Tetzlaff, P. Manoonpong, Fast dynamical coupling enhances frequency adaptation of oscillators for robotic locomotion control, Frontiers in Neurorobotics 11.

[33] T. Petrič, A. Gams, A. J. Ijspeert, L. Žlajpah, On-line frequency adaptation and movement imitation for rhythmic robotic tasks, The International Journal of Robotics Research 30 (14) (2011) 1775-1788.

[34] G. Chen, H. Yu, Adaptive-oscillator-based control strategy for gait rehabilitation robots, in: Converging Clinical and Engineering Research on Neurorehabilitation II, Springer, 2017, pp. 1365-1369. 
[35] K. Seo, S. Hyung, B. K. Choi, Y. Lee, Y. Shim, A new adaptive frequency oscillator for gait assistance, in: Robotics and Automation (ICRA), 2015 IEEE International Conference on, IEEE, 2015, pp. 5565-5571.

[36] L. Peternel, T. Noda, T. Petrič, A. Ude, J. Morimoto, J. Babič, Adaptive control of exoskeleton robots for periodic assistive behaviours based on emg feedback minimisation, PloS one 11 (2) (2016) e0148942.

[37] L. Righetti, A. J. Ijspeert, Pattern generators with sensory feedback for the control of quadruped locomotion, in: Proceedings of International Conference on Robotics and Automation (ICRA) 2008, 2008, pp. 819-824.

[38] L. Righetti, J. Buchli, A. J. Ijspeert, Dynamic hebbian learning in adaptive frequency oscillators, Physica D: Nonlinear Phenomena 216 (2) (2006) 269-281.

[39] L. Righetti, J. Buchli, A. J. Ijspeert, Adaptive frequency oscillators and applications, Open Cybernetics \& Systemics Journal 3 (2009) 64-69.

[40] M. N. Mahdi Khoramshahi, Auke Ijspeert, Exploiting natural dynamics of nonlinear compliance using adaptive oscillators, in: Adaptive Motion of Animal and Machine 2013, 2013

[41] MATLAB, Mathworks Inc. URL http: //www. mathworks. com

[42] M. H. Raibert, Legged robots that balance, Vol. 3, MIT press Cambridge, MA, 1986.

[43] O. Khatib, A unified approach for motion and force control of robot manipulators: The operational space formulation, Robotics and Automation, IEEE Journal of 3 (1) (1987) 43-53.

[44] QUANSER, Rotary flexible joint (2014).

URL http://www.quanser.com/Products/rotary_flexible_joint

[45] F. Nori, Stiction compensation in agonist-antagonist variable stiffness actuators.

[46] H.-B. Shin, New antiwindup pi controller for variable-speed motor drives, IEEE Transactions on Industrial Electronics 45 (3) (1998) $445-450$.

[47] M. Khoramshahi, A. Parsa, A. Ijspeert, M. N. Ahmadabadi, Natural dynamics modification for energy efficiency: A data-driven parallel compliance design method, in: 2014 IEEE International Conference on Robotics and Automation (ICRA), IEEE, 2014, pp. $2412-2417$.

[48] R. Nasiri, M. Khoramshahi, M. Shushtari, M. N. Ahmadabadi, Adaptation in variable parallel compliance: Towards energy efficiency in cyclic tasks, IEEE/ASME Transactions on Mechatronics 22 (2) (2017) 1059-1070.

[49] R. Nasiri, M. Khoramshahi, M. N. Ahmadabadi, Design of a nonlinear adaptive natural oscillator: Towards natural dynamics exploitation in cyclic tasks, in: 2016 IEEE/RSJ International Conference on Intelligent Robots and Systems (IROS), IEEE, 2016, pp.--

[50] S. Schaal, Dynamic movement primitives-a framework for motor control in humans and humanoid robotics, in: Adaptive Motion of Animals and Machines, Springer, 2006, pp. 261-280.

[51] E. Theodorou, J. Buchli, S. Schaal, Learning policy improvements with path integrals, in: International Conference on Artificial Intelligence and Statistics, 2010, pp. 828-835.

[52] M. Ajallooeian, M. N. Ahmadabadi, B. N. Araabi, H. Moradi, Design, implementation and analysis of an alternation-based central pattern generator for multidimensional trajectory generation, Robotics and Autonomous Systems 60 (2) (2012) 182-198.

[53] M. Ajallooeian, J. van den Kieboom, A. Mukovskiy, M. A. Giese, A. J. Ijspeert, A general family of morphed nonlinear phase oscillators with arbitrary limit cycle shape, Physica D: Nonlinear Phenomena 263 (2013) 41-56.

[54] M. W. Spong, S. Hutchinson, M. Vidyasagar, Robot modeling and control, Vol. 3, Wiley New York, 2006. 